\title{
Philosophiques
}

\section{Avortement, éthique sociale et positivisme juridique}

\section{François Blais}

Volume 18, numéro 2, automne 1991

URI : https://id.erudit.org/iderudit/027152ar

DOI : https://doi.org/10.7202/027152ar

Aller au sommaire du numéro

Éditeur(s)

Société de philosophie du Québec

ISSN

0316-2923 (imprimé)

1492-1391 (numérique)

Découvrir la revue

Citer cet article

Blais, F. (1991). Avortement, éthique sociale et positivisme juridique. Philosophiques, 18(2), 63-78. https://doi.org/10.7202/027152ar

\section{Résumé de l'article}

Cet article a pour objectif de défendre un point de vue positiviste du droit de certaines erreurs et confusions commises à son égard. L'auteur se sert d'un récent article de G. Legault ( "La parole du philosophe éthicien est-elle crédible? ») et reprend certaines affirmations identifiant le mouvement pro-choix à la doctrine positiviste. L'auteur tente de démontrer que ce rapprochement, pour l'essentiel, ne tient pas et qu'il repose sur une méconnaissance du positivisme juridique contemporain. En dernière partie, l'auteur discute de certains intérêts théoriques et pratiques d'une conception plus large du concept de droit telle que privilégiée par le positivisme juridique. 


\title{
AVORTEMENT, ÉTHIQUE SOCIALE ET POSITIVISME JURIDIQUE*
}

\author{
par François Blais
}

REsumE. Cet article a pour objectif de défendre un point de vue positiviste du droit de certaines erreurs et confusions commises à son égard. L'auteur se sert d'un récent article de G. Legault ("La parole du philosophe éthicien est-elle crédible? ») et reprend certaines affirmations identifiant le mouvement pro-choix à la doctrine positiviste. L'auteur tente de démontrer que ce rapprochement, pour l'essentiel, ne tient pas et qu'il repose sur une méconnaissance du positivisme juridique contemporain. En dernière partie, l'auteur discute de certains intérêts théoriques et pratiques d'une conception plus large du concept de droit telle que privilégiée par le positivisme juridique.

ABSTRACT. This article aims to defend a positivist approach of Law against some misconceptions about it. The author uses a recent article of G. Legault ("La parole du philosophe éthicien est-elle crédible? ") to discuss some assumptions about legal positivism and the pro-choice movement for abortion. $\mathrm{He}$ criticizes the point of view that some similarities between these two doctrines exist and he attempts to demonstrate that those assumptions are the result of many confusions about the legal positivism and the pro-choice movement. Finally, the author discusses about some fundamental reasons, theoretical and pratical, to prefer the legal positivism doctrine of Law to naturalism.

* Je tiens à remercier pour leurs précieux commentaires Pierre-Yves Bonin, Nicolas Kaufmann et Georges Leroux. Je remercie aussi pour son appui financier le Conseil de recherches en sciences humaines du Canada qui a subventionné une recherche doctorale sur la philosophie de l'action chez H. L. A. Hart et qui subventionne maintenant une recherche postdoctorale sur les droits fondamentaux et le naturalisme juridique. 


\section{Introduction}

La question du rapport entre droit et moralité est au nombre des perplexités centrales retenues par l'histoire de la philosophie du droit jusqu'à nos jours. Dans la lignée des discussions nombreuses sur ce sujet de prédilection de la philosophie du droit, j'aimerais dans ce texte défendre une approche particulière de ce problème, le positivisme juridique, contre certaines erreurs et confusions fréquemment commises à son endroit. Ces erreurs et confusions dissipées, on devrait, je l'espère, être plus en mesure d'apprécier à sa juste valeur cette doctrine du droit ainsi que les éclaircissements qu'elle apporte sur ce problème fondamental du rapport entre droit et moralité.

Plusieurs arguments ont été invoqués à la défense de la thèse positiviste. Pour ma part, je défendrai principalement l'idée que les motifs qui devraient nous faire pencher du côté du positivisme juridique plus que du côté du naturalisme sont en dernière instance de nature morale. Une telle affirmation semblera à plusieurs en contradiction avec leur propre conception du positivisme en droit, conception selon laquelle cette doctrine favoriserait grossièrement la primauté de l'étude du droit positif et de la méthodologie propre à son examen sur toute considération philosophique et rechercherait ultimement à affranchir le droit positif de toute influence morale ${ }^{1}$. Ce malentendu sur la nature du positivisme juridique est malheureusement trop répandu et il demande à être éclairci avant toute chose pour ne pas que l'on réduise ce dernier à ce qu'il n'est pas: une sorte de méthodologie positive de la "science juridique".

Les confusions que je reproche aux philosophes et au milieu intellectuel en général de commettre à l'égard du positivisme juridique seront illustrées à partir d'un article récent de G. Legault ${ }^{2}$. Dans cet article, Legault cherche à établir les balises de la tâche à venir de l'éthicien. L'auteur en vient à conclure à l'importance de redéfinir radicalement le point de vue que se donne la philosophie morale de sa tâche afin de tourner le dos aux approches fondationnelles en éthique et de s'investir plutôt dans des préoccupations tournées vers la nature de la délibération et du "partage des sens", la nature du développement moral et la recherche de corroboration par les disciplines des sciences humaines du contenu des discours des éthiciens. Je ne discuterai pas ici de l'essentiel de ces idées, que je considère d'un grand intérêt par ailleurs, ni même d'idées proches voisines 
ou connexes. Mon attention se limitera à un passage du texte de Legault ayant trait au positivisme juridique. Dans ce passage ${ }^{3}$, Legault établit certains rapprochements entre la rationalité du mouvement pro-choix en matière $d^{\prime}$ avortement et la doctrine positiviste. Je compte me servir de ce passage du texte de Legault pour illustrer ce qui selon moi fait partie d'erreurs fréquemment commises à propos du positivisme juridique et peut-être aussi à l'égard du mouvement pro-choix.

J'estime que Legault, dans ce passage sur les rapports entre le mouvement pro-choix et le positivisme juridique, commet trois erreurs. Il s'agit de:

(a) Associer à une forme de positivisme juridique la revendication du mouvement pro-choix de maintenir une certaine autonomie du droit positif à l'égard de toute conception morale ou religieuse particulière.

(b) Concevoir le positivisme juridique comme opposant deux ordres de normativité: l'un moral et l'autre juridique; ou encore affirmer que l'autonomie revendiquée en (a) a pour conséquence cette opposition.

(c) Affirmer que la philosophie positiviste du droit, dans la mesure où elle associe "la morale sociale aux moeurs [...] n'ouvre aucune dimension à la délibération sociale, à une éthique de la délibération puisque les moeurs sont un produit historique ${ }^{4}$.

Puisque ces trois erreurs reposent pour l'essentiel sur une certaine conception erronée du positivisme en droit, je présenterai (Partie I) une définition des principales caractéristiques du positivisme juridique contemporain et je montrerai par la suite (Partie II) en quoi selon moi certaines affirmations de Legault portées à l'endroit du positivisme juridique me semblent fausses. Je terminerai mon intervention (Partie III) en identifiant de quelle manière le positivisme juridique, tout en favorisant une compréhension plus adéquate de la nature du droit, est aussi en mesure de susciter plus que toute autre doctrine du droit une attitude plus positive à l'égard de la place que devrait occuper la délibération éthique dans une société démocratique. Il est à souhaiter que dans un avenir prochain, les débats trop souvent doctrinaires et sans issue opposant les deux camps face à l'avortement puissent tirer profit de telles dispositions d'esprit. 


\section{LA SÉPARATION CONCEPTUELLE DU DROIT ET DE LA MORALITÉ: LE POSITIVISME JURIDIQUE}

La controverse entourant la relation qu'entretiennent droit et moralité représente très certainement un sujet de prédilection de la littérature classique en philosophie du droit. On ne s'étonnera donc pas de constater qu'à travers les époques, cette controverse a revêtu différentes formes, au point où il est permis de se questionner aujourd'hui sur le sens profond des divergences en cause $e^{5}$. Il est donc essentiel, sur ces questions opposant positivisme et naturalisme, de se doter tout d'abord d'une définition claire de ce qu'on entend par l'expression "positivisme juridique » afin d'éviter justement de s'embourber dans des querelles de mots et d'interprétation des théories classiques ou même contemporaines.

Le point de départ que j'utiliserai dans cette intervention est emprunté à l'un des philosophes du droit contemporain les plus importants: Herbert Hart ${ }^{6}$. Hart est considéré à juste titre comme un représentant majeur du positivisme juridique et comme le principal responsable au $X X^{\mathrm{e}}$ siècle, avec $\mathrm{H}$. Kelsen, du renouveau d'intérêt pour cette doctrine du droit. La position initiale retenue par Hart pour définir son propre positivisme juridique emprunte par ailleurs directement aux conceptions développées un siècle auparavant par les grands philosophes du droit que furent J. Austin et J. Bentham. À la suite de ses prédécesseurs positivistes, Hart défend la thèse centrale qu'entre moralité et droit positif, il y a absence de lien conceptuel nécessaire:

Nous utiliserons ici l'expression " positivisme juridique » pour désigner la simple thèse selon laquelle il n'est en aucune manière nécessairement vrai que les règles de droit reflètent ou donnent satisfaction à certaines exigences morales, bien qu'en réalité elles l'aient souvent fait?

Grosso modo, la thèse naturaliste depuis Aristote, Thomas d'Aquin, Locke et Kant soutient, contrairement au positivisme, que le droit positif dérive au moins partiellement de principes moraux autonomes et dont la légitimité se trouve soit dans un certain ordre métaphysique (Aristote et Thomas d'Aquin), soit dans le contenu de réflexions spéculatives sur la nature de l'être humain et de la vie en société (Locke et Kant). Il existe ainsi, selon les tenants du naturalisme, un "lien " entre droit et moralité qu'il est nécessaire d'expliciter pour comprendre véritablement la "nature " du droit. 
La thèse positiviste, telle que défendue par Hart et d'autres, nie que le droit soit essentiellement moral et affirme au contraire que l'existence du droit est un problème conceptuellement distinct de son mérite ou son démérite moral. Essentiellement, la question de ce qu'est le droit doit pouvoir se poser séparément de la question de savoir ce qu'il devrait être et on ne peut pas, comme le prétendent les tenants du droit naturel, refuser le statut de "loi " à une règle légale jugée "mauvaise". Le rapport droit-moralité est pour l'essentiel un rapport contingent.

Hart a produit une longue et minutieuse argumentation pour venir à bout de la thèse naturaliste. Sa position a aussi évolué avec le temps ${ }^{8}$. Je ne veux pas la discuter en détail ici mais je crois cependant pouvoir résumer les composantes essentielles de son positivisme à l'intérieur des quatre affirmations qui suivent:

$P_{1}$ Le positivisme juridique est une thèse indépendante d'autres thèses que l'on a pu rattacher traditionnellement aux positivistes (que ce soit par exemple d'être tenants et défenseurs: (i) de la méthodologie de la théorie générale du droit (la "jurisprudence" des anglo-saxons); (ii) de la théorie impérativiste du droit (cf. le théoricien du droit du XIXe siècle, J. Austin); (iii) du formalisme juridique ou encore (iv) du relativisme en éthique) ${ }^{9}$.

$P_{2}$ La thèse positiviste ne nie pas: (i) le fait historique que droit et moralité s'influencent mutuellement; (ii) que des considérations morales puissent être prises en compte pour décider de ce que le droit devrait être et pour dénouer une impasse lors de conflits d'interprétation de règles particulières. Elle rejette seulement la conception du droit voulant qu'une loi «injuste" ne soit pas une $\operatorname{loi}^{10}$.

$\mathrm{P}_{3}$ Le positivisme juridique n'est pas lié à un type d'obligation morale inconditionnelle d'obéissance à la loi ${ }^{11}$.

$\mathrm{P}_{4}$ Le positivisme $n^{\prime}$ interdit pas que l'on puisse reconnaître l'existence pour toute forme de système légal d'un «contenu minimum de droit naturel » qui équivaut à des règles de droit assurant un minimum de protection physique et de survie aux membres d'une collectivité. À ces règles de survie on peut ajouter éventuellement deux règles formelles: l'impartialité et l'objectivité. Ce "minimum de droit naturel", par ailleurs, n'équivaut pas à ce qui fut habituellement reconnu comme étant du «droit naturel » par les partisans du naturalisme ${ }^{12}$. 
Ces quatre thèses constituent l'essentiel du positivisme juridique de Hart. Nous croyons aussi qu'elles représentent assez correctement la tradition positiviste contemporaine dans l'ensemble. Cependant, malgré leur cohérence et leur valeur explicative, ces thèses ne fournissent pas à elles seules les motifs pour lesquels il est en dernière analyse plus raisonnable d'adhérer au positivisme plutôt qu'au naturalisme. Ces motifs pourtant existent et ils seront considérés à la fin de notre texte. Nous comptons pour le moment revenir sur les liens présumés entre le mouvement pro-choix et le positivisme. Les éléments mis en place jusqu'ici devraient être suffisants pour passer au premier examen des principales interrogations soulevées par le texte de Legault à propos du positivisme juridique et $\mathrm{du}$ mouvement pro-choix.

\section{LES ARGUMENTS DU MOUVEMENT PRO-CHOIX EN FAVEUR DE L'AVORTEMENT ET LE POSITIVISME JURIDIQUE: DES QUESTIONS DISTINCTES}

\section{A. Autonomie du droit et positivisme juridique}

La première erreur du texte de Legault est d'avoir identifié comme participant à une conception positiviste du droit la revendication, par ailleurs maintes fois répétées par les militantses du mouvement pro-choix, selon laquelle il faut chercher à protéger le droit positif de toute appartenance à une conception religieuse particulière ou encore à un code moral particulier. Cette revendication, comme on le sait, a trouvé l'une de ses formulations célèbres dans la déclaration "La religion des uns ne doit pas devenir la loi des autres " entendue lors de la dernière campagne électorale au Québec.

Il y aurait beaucoup à dire sur ce seul aspect du problème. Je me contenterai d'identifier la méprise qui me semble la plus importante: il s'agit de prétendre, ou du moins de laisser entendre comme le fait je crois Legault, que le contenu de cette revendication du mouvement pro-choix veut éloigner notre code légal en matière d'avortement de toute conception morale. La chose apparaît plutôt curieuse à première vue. Si on considère l'ensemble des règles du droit positif d'une société, on doit convenir qu'il est impensable de formuler de telles règles sans, d'une manière ou de l'autre, refléter certaines valeurs morales dont sont issues justement ces règles de droit ${ }^{13}$. Le mouvement pro-choix quand il cherche à défendre sa position en faveur de l'accès libre et gratuit à l'avortement n'échappe 
pas à cette situation de fait et les présuppositions morales qui orientent nécessairement le contenu du droit positif ne lui sont certainement pas indifférentes. On invoquera, par exemple, la capacité morale de la mère de décider "en son âme et conscience " du sort du foetus qu'elle porte et de la supériorité de ce droit individuel sur tout autre principe ayant trait par exemple aux "statuts et privilèges » de ce foetus, aux "droits du père ", etc. La position pro-choix n'est ainsi formellement pas plus dépourvue de considérations de nature morale que ne l'est la position pro-vie. La question qui se pose dans le cas de l'avortement comme dans le cas de toute situation où le contenu de notre droit positif est en question ou est source de conflit est de savoir quel contenu normatif doit encadrer nos règles de droit et sur quels fondements peut-il s'appuyer? ${ }^{14}$.

Les militants du mouvement pro-choix n'échappant pas aux présuppositions morales du droit positif, il faut donc présumer à leur défense qu'ils entendent quelque chose de particulier par la revendication de la séparation claire du droit et de la morale. Dans l'objectif d'accorder une certaine consistance à leur propos, on doit postuler qu'ils désirent plutôt voir s'établir une séparation claire entre le droit positif et certaines conceptions morales et surtout religieuses qui revendiquent, par exemple, le droit (naturel) à la vie du foetus. La revendication du mouvement pro-choix de tenir à l'écart certaines considérations religieuses du contenu du droit positif en matière d'avortement ne doit donc pas être interprétée comme une volonté de nier toute signification morale au droit en général, ce qui est, je le répète, un non-sens, mais plutôt comme le souhait d'éliminer les conceptions morales ou religieuses apparaissant plus menaçantes vis-à-vis de la cause que ses partisans défendent.

Mais il faut ici faire les nuances qui s'imposent. On peut probablement affirmer sans se tromper que sur les questions liées à l'avortement, le mouvement pro-choix est en général plutôt en faveur d'un contenu "laïcisé » du code légal qui aurait comme avantage, eu égard à leur position, d'évacuer des débats législatifs en matière de droit à l'avortement toute discussion portant sur la "nature» de l'être humain ou sur les "droits » de l'embryon ou du foetus ainsi que, d'une façon plus générale, de maintenir plus ouvert le champ des libertés individuelles et de diminuer les opportunités pour une conception religieuse ou morale particulière d'imposer ses propres valeurs à l'ensemble de la société par le biais du droit criminel. Ce 
parti-pris reconnu, on aurait tort cependant de l'assimiler au positivisme, bien qu'il faille admettre que certains propos émanant du mouvement pro-choix puissent avoir contribué à entretenir cette confusion. Philosophiquement, un tel rapprochement ne tient pas. Une perspective philosophique sur le droit comme le positivisme juridique n'a rien à voir avec la conception « laïcisé » du droit à l'avortement des partisans du mouvement pro-choix qui n'est en fait, à la regarder de près, qu'une position stratégique visant à écarter du contenu du droit des conceptions morales et religieuses particulières. Il n'est a priori pas plus interdit pour un partisan du libre-choix de défendre un naturalisme strict qu'il ne lui est interdit d'adopter une perspective positiviste. On peut très bien, pour donner un exemple, envisager l'hypothèse d'un partisan pro-choix naturaliste qui prétend que les raisons de son parti-pris pour l'accès libre à l'avortement reflètent «l'essence » même du droit dans une société libérale et démocratique et qui considère pour cette raison et d'autres qu'on ne peut l'empêcher et encore moins le criminaliser.

Si le rapprochement que plusieurs sont tentés de faire entre le positivisme juridique et le libre-choix à l'avortement ne tient pas, peut-on en dire autant du rapprochement que d'autres croient légitime d'établir entre le naturalisme et le mouvement pro-vie? Répondre par l'affirmative à cette question serait encore une fois prendre des conjonctures pour des nécessités. Il est vrai que les faits à notre disposition indiquent une tendance très forte des militants pro-vie à défendre leur position à partir d'une tradition religieuse qui, la plupart du temps, établit un rapport continu et complémentaire entre moralité (religieuse) et droit, ce qui fait pencher de facto ces militants pro-vie du côté d'un certain naturalisme en droit. L'erreur toutefois serait d'en tirer comme conclusion que tout partisan pro-vie est irrémédiablement naturaliste ou que la doctrine naturaliste entraîne nécessairement ceux qui la défendent à s'opposer à l'avortement. Il y a en fait des exemples célèbres montrant que tel $n^{\prime}$ est pas toujours le cas $^{15}$. Il n'existe selon moi aucune relation conceptuelle permettant de lier définitivement l'un à l'autre. L'éventualité d'un positiviste pro-vie est aussi envisageable que celle d'un naturaliste pro-choix qui considèrerait par exemple que le fotus avant un certain âge est un "nonêtre » ou encore qu'il est dépourvu de tout statut juridique.

En conclusion sur ce premier point: l'objectif prêté par Legault au mouvement pro-choix de vouloir dissocier le contenu 
du droit de toute référence à un ordre moral ou religieux, s'il était réalisable, n'emprunte rien au positivisme juridique. Le positivisme juridique ne nie pas ce fait évident qu'il y a une influence de la morale sur le choix de nos contenus légaux tout comme il y a une influence du droit sur la morale (cf. $\mathrm{P}_{2}$ ). Il ne nie pas non plus l'importance et la valeur des actions de ceux qui cherchent à améliorer notre droit positif dans le but de le rendre plus légitime et plus conforme à nos aspirations de justice, bien au contraire.

\section{B. La soi-disant opposition entre normativité morale et normativité juridique}

À la suite de ce que je viens d'affirmer sur l'influence réciproque de la moralité et $\mathrm{du}$ droit, j'aimerais ajouter que ce serait commettre une erreur tout aussi importante de prétendre que toute tentative de distinguer conceptuellement le droit et la moralité conduise nécessairement à l'opposition de ces deux ordres de normativité. Je crois pour ma part que l'idée « d'opposition » est trompeuse ici et qu'elle a tendance à créer une autre confusion sur la nature du positivisme juridique ou encore sur la portée de la revendication pro-choix vue en A.

Il est tout d'abord évident que les faits en cette matière corroborent l'existence de deux ordres de normativité. En effet, que l'on soit partisan du positivisme ou du naturalisme, on admet en général que, formellement, règles légales et règles morales diffèrent sur plusieurs plans. Leur contexte de production et de justification, les mécanismes qui en assurent la transformation, leur statut, leur degré "d'objectivité » ou encore la nature des "conséquences" rattachées à leur infraction constituent tous des éléments qui justifient largement que l'on distingue ces ordres de normativité et que l'on s'attache à en clarifier les différences. L'éclaircissement de ces différences est d'ailleurs au centre de la tâche que s'est donnée la théorie générale du droit depuis Bentham et Austin ${ }^{16}$. Celles-ci étant déjà pour une large part reconnues, il ne faudrait pas pour autant traduire en terme "d'opposition" le rapport entre ces ordres de normativité, surtout si on entend par "opposition" un rapport d'exclusion simple entre le contenu normatif des règles de droit d'une société particulière et le contenu des multiples règles morales que l'on retrouve en son sein. Distinguer la nature des règles de droit de la nature des règles morales est une chose qui tombe aujourd'hui sous le coup de l'évidence et le professeur 
Legault par son ouvrage sur La structure performative du langage juridique est là pour en témoigner ${ }^{17}$. Je ne crois pas, par ailleurs, que le positivisme juridique, en revendiquant une séparation conceptuelle claire entre droit et morale, affirme du même coup que ces ordres normatifs s'excluent ou qu'ils devraient s'exclure. L'erreur à ne pas commettre, et cela le positivisme juridique, $s^{\prime}$ en garde, serait de nier justement les influences réciproques réelles ou encore à venir entre les orientations normatives des deux types de règles en cause, ce qui de toute façon serait immédiatement pris en défaut par l'histoire et la sociologie du droit. Encore une fois ici, le contenu de l'affirmation $\mathrm{P}_{2}$ plus haut, selon laquelle le positivisme reconnait l'influence réciproque du contenu du droit et de la moralité, témoigne clairement de sa position sur ces questions. Quant au mouvement pro-choix, il faut comprendre encore une fois que l'objectif recherché en réclamant une certaine autonomie du droit par rapport à la morale ne peut en aucune façon être identifié ou même associé à celui du théoricien du droit positiviste qui, pour sa part, ne vise qu'à favoriser une meilleure compréhension de la nature du droit.

\section{La délibération des valeurs et le positivisme juridique}

Ce que nous avons vu jusqu'ici devrait nous conduire à une dernière méprise sur le positivisme juridique. Legault dans son texte affirme: «Dans la mesure où certains membres du mouvement pro-choix s'inspirent de la philosophie positiviste $\mathrm{du}$ droit, ils identifient la morale sociale aux moeurs. Une telle conception n'ouvre aucune dimension à la délibération sociale puisque les moeurs sont un produit historique ${ }^{18}$. L'erreur ici est de sous-entendre que le positivisme juridique tend à limiter les discussions et les délibérations sur le contenu des règles légales. A ce propos, est-il nécessaire de rappeler que le positivisme d'aucune façon ne doit être vu comme une caution théorique liant les individus d'une société à une sorte d'obéissance inconditionnelle (cf. $P_{3}$ )? J'ajouterais même que dans le cas du positivisme juridique, c'est plutôt la réaction contraire qui est attendue et que cela devrait être une raison supplémentaire, sinon définitive, pour préférer le positivisme juridique au naturalisme qui, lui, tend peut-être davantage à limiter la perspective de la délibération morale si importante et nécessaire aux vues de Legault. Mais pour montrer cela, nous devons revenir à Hart et aux arguments et avantages qui devraient rationnellement nous amener à souscrire au positivisme juridique. 


\section{POSITIVISME JURIDIQUE ET DÉLIBÉRATION ÉTHIQUE}

La motivation principale du positivisme comme Hart le conçoit n'est certainement pas de chercher de quelque façon que ce soit à immuniser le droit afin de le rendre indifférent à toute discussion éthique. Les arguments qui plaident en sa faveur sont tout d'abord d'ordre cognitif et épistémologique. En effet, en n'enlevant pas a priori le statut de règles légales à des règles de droit qui, bien qu'appartenant à un système législatif reconnu, peuvent apparaître à certains ou même à plusieurs immorales, le positivisme permet la constitution d'un objet théorique moins évanescent et plus stable pour la théorie générale du droit. L'apport des théoriciens du droit moderne ne pourrait pas être ce qu'il est devenu aujourd'hui s'il avait été impossible d'étudier le droit pour ce qu'il est et de la façon dont il se présente dans une société, et cela sans se préoccuper continuellement de la question de savoir si ce droit particulier est juste ou non. Sans cette première préoccupation pour une définition "élargie» du concept de droit, l'objet même d'étude de la théorie générale du droit moderne resterait pratiquement insaisissable et, pour cette raison, dépourvu d'intérêts théoriques.

Mais il y a un deuxième argument qui justifie, une fois reconnues les préoccupations cognitives ou épistémologiques permettant la construction d'un objet d'étude, d'endosser le point de vue positiviste du droit. Celui-ci est de nature morale:

Mais il y a peut-être une raison plus péremptoire de préférer le concept de droit le plus large qui nous permettra de penser et de dire "C'est du droit mais il est inique »: c'est que le refus de reconnaître le caractère juridique de règles iniques peut constituer une simplification excessive de l'éventail de questions morales qu'elles soulèvent. Des auteurs plus anciens qui comme Bentham et Austin, ont insisté sur la distinction entre ce que le droit est et ce qu'il doit être, ont agi ainsi partiellement parce qu'ils pensaient qu'à moins d'opérer cette distinction, les hommes pourraient, sans tenir compte de leur coût pour la société, émettre des jugements hâtifs concluant à la non-validité des règles de droit et à l'absence de devoir d'obéissance ${ }^{19}$.

Le naturalisme peut conduire à deux types d'excès diamétralement opposés et largement condamnables. Le premier de ces excès est l'anarchisme, tel qu'indiqué par Hart dans la citation plus haut. Une caractérisation naturaliste et restreignante du droit est, croit-on, plus propice à conduire à l'anarchisme 
puisque dans toute situation particulière où nous sommes confrontés personnellement ou collectivement avec un droit ou une obligation, le naturalisme permettrait toujours le recours à un faux-fuyant du type: "nous n'avons qu'à juger « immoral », individuellement ou collectivement, ce droit ou cette obligation pour ne pas nous sentir obligé de nous y soumettre ". L'usage d'un tel faux-fuyant conduit de manière aveugle à la désobéissance civile et surtout ne permet pas à celui qui y recourt de réfléchir convenablement sur des motifs plus sérieux qui devraient pouvoir justifier autrement son geste. À l'opposé de l'anarchisme se trouve un autre excès: le conservatisme extrême. En effet, une conception restreinte du concept de droit telle celle du naturalisme peut servir de prétexte à ceux qui se serviront de la maxime: "si tout droit doit être moral, tout droit doit être moralement engageant " pour préserver le plus longtemps possible le statu quo et empêcher les réformes justifiées de s'accomplir.

Parce que les questions de l'obéissance et de la désobéissance à la loi sont des questions sérieuses, d'une grande complexité et qui ne doivent pas être écartées au moment où elles se posent à un individu ou à un groupe, on doit probablement maintenir la conception la plus large d'une règle de droit au détriment $d^{\prime}$ une conception plus restrictive comme dans le cas du naturalisme. Une conception restrictive du concept de droit peut fournir l'occasion à des individus membres d'une société démocratique d'éviter d'examiner dans toute leur dimension morale les problèmes spécifiques auxquels une règle de droit tente de répondre, tout comme de réfléchir convenablement sur les conséquences de leurs décisions de décréter péremptoirement la non-validité d'une loi particulière. Pour éviter ces excès de l'anarchisme et du conservatisme extrême qui constituent des maux pour les exigences pratiques de la vie en société démocratique et pour la décision morale en particulier, il faut probablement accepter de prendre une attitude plus ouverte à l'égard du droit que ne le fait le naturalisme. Cette ouverture à l'égard du droit ne nous interdit pas, par ailleurs, d'adopter des points de vue radicaux si les circonstances nous y obligent:

La chose qui est de loin la plus nécessaire pour rendre les gens plus clairvoyants lorsqu'ils sont en présence de l'abus d'un pouvoir d'autorité, c'est qu'ils gardent la conscience de ce que l'assurance de la validité juridique n'est pas décisive quant au problème de l'obéissance, et que, quelle que soit la dimension de l'aura de majesté ou d'autorité que puisse avoir 
le système officiel, ses exigences doivent finalement être soumises à un examen moral minutieux ${ }^{20}$.

Cette motivation profonde du positivisme est selon moi fondamentale et elle est révélatrice de son adhésion aux traditions les plus nobles et les plus libérales de nos sociétés démocratiques. Elle fait du positivisme une doctrine aux préoccupations pratiques et morales égales sinon supérieures aux doctrines naturalistes traditionnelles, contrairement à une fausse perception largement répandue qui voudrait faire du positivisme juridique une sorte de justification théorique du statu quo, un prétexte à l'immobilisme.

La motivation pratique qui justifie le positivisme juridique s'inscrit en dernier lieu dans un objectif plus général de contribuer à éduquer le citoyen à l'usage d'un concept de droit le plus large qui soit afin d'augmenter sa capacité de réfléchir de manière critique sur ce même droit ainsi que sur le pouvoir qui le sanctionne. Pour atteindre cet objectif, il faut probablement accepter le principe positiviste voulant que toute règle formulée adéquatement et remplissant certains critères formels puisse être reconnue comme valide, et cela indépendamment du fait qu'elle soit ou non moralement acceptable pour un groupe particulier, afin que tout citoyen ait l'occasion de se confronter à son application et qu'il puisse éventuellement entretenir à son égard une réflexion morale continue et non dogmatique. Comme il n'y a pas de fondement conceptuel suffisant pour supposer que le droit qui est, est nécessairement le droit qui devrait être courant, le positivisme sur ce problème soutient que le droit reste toujours le plus ouvert possible à la critique morale.

\section{Conclusion}

Le positivisme juridique, loin de défendre une position conservatrice, s'attache plutôt à défendre une perspective lucide et essentiellement critique à l'égard de l'autorité du droit et de la morale. Il offre un soutien supplémentaire à la délibération éclairée. Une telle perpective par rapport au droit est préférable à toute autre, particulièrement dans un contexte comme celui des débats sur l'avortement où l'émotivité et le radicalisme l'emportent trop souvent sur la discussion rationnelle, éclairée et non dogmatique.

Pour revenir aux thèses de Legault, si j'ai bien raison au sujet des trois erreurs que je lui attribue, je crois que ce dernier est pris dans un dilemme. $S^{\prime} i l$ tient malgré tout à rattacher 
au mouvement pro-choix ou encore à certains de ses membres l'étiquette de "positivistes juridiques ", il sera obligé en retour de soupeser sérieusement l'idée que j'ai voulu défendre ici, qu'en regard de la reconstruction de la tâche éthique dont il dresse les grandes orientations dans son article, le fait d'être positiviste favorise davantage la délibération éthique éclairée et non dogmatique que le naturalisme ne peut le faire. Le mouvement pro-choix partirait donc, suivant ce point de vue et contrairement aux idées de Legault, avec une longueur d'avance sur toute autre position s'attachant à défendre en arrière-fond de son argumentation une perspective naturaliste du droit.

\section{Centre de recherche en droit public \\ Université de Montréal}

Département de philosophie

Université de Toronto

\section{NOTES}

1. Cette attitude à l'égard du positivisme juridique est présente par exemple dans un texte du professeur Guy Durand paru pour la première fois en 1986 dans la revue Thémis. L'auteur y défini le positivisme et le naturalisme juridique: «La première tendance - positiviste - tend à calquer la loi sur les moeurs ou l'opinion publique: La loi ne devrait être que la codification de celle-ci et évoluer au même rythme. Le droit serait indépendant, voire opposé à la morale. L'autre tendance - naturaliste - rattache davantage le droit à la morale, parfois jusqu'à les identifier. Le droit ayant un rôle normatif, il devrait découler d'une réflexion rationnelle sur les valeurs ». Cette citation est tirée d'un extrait de cet article paru en 1989 dans la revue L'Analyste, vol. 27, p. 51. Je souligne.

2. Georges A. Legault: "La parole du philosophe éthicien est-elle crédible? ", Philosophiques, vol. 27, numéro 1 (Printemps 1990), pp. 21-43.

3. Voir pp. 31-32 du texte de Legault.

4. Ibid. p. 32 .

5. Robert S. Summers: «The New Analytical Jurists », New York University Law Review, vol. 41 (1966), p. 889 dénombre pas moins de dix définitions différentes du positivisme juridique à travers le temps. Elles ne sont cependant pas toutes d'un intérêt égal.

6. C'est dans "Positivism and the Separation of Laws and Morals", Harvard Law Review, vol. 71 (1958), pp. 593-629 que Hart défend 
pour la première fois son point de vue positiviste du droit. $\mathrm{Ce}$ texte est repris dans son Essays in Jurisprudence and Philosophy, Oxford: Clarendon Press, 1983, pp. 49-87. On doit aussi lire à ce sujet son The Concept of Law, Oxford University Press, première édition 1961, 263 p. au chap. IX: "Laws and Morals".

7. Le concept de droit (traduction française par Michel Van de Kerchove) Bruxelles: Facultés Universitaires Saint-Louis, 1976, p. 224.

8. On n'a qu'à rappeler ici le débat opposant Hart à Lon L. Fuller. Ce dernier, représentant important de la doctrine du droit naturel, a critiqué le positivisme de Hart dans sa première version de1958, à l'intérieur d'un article intitulé: " Positivism and Fidelity to LawA Reply to Professor Hart ", Harvard Law Review, vol. 71 (1958), pp. 630-72 et dans un ouvrage de 1964: The Morality of Law, New Haven: Yale University Press, 262 p. Le débat s'est prolongé dans un compte rendu critique de Hart du livre de Fuller: « Review of The Morality of Law by Lon Fuller ", Harvard Law Review, vol 78 (1965), pp. 1281-96 et dans une édition ultérieure de The Morality of Law (1969) où Fuller ajoute un 5e chapitre: "A Reply to Critics". M. Martin dans The Legal Philosophy of H.L.A. Hart: A Critical Appraisal, Philadelphia: Temple University Press, 1987, pp. 209-37 fait un excellent compte rendu de ce débat majeur et reconnaît, comme d'autres commentateurs d'ailleurs, que Hart a relativement bien disposé des arguments de Fuller.

9. Sur ce point voir Hart: "Legal Positivism " dans Paul Edwards (ed.): The Encyclopedia of Philosophy, New York: Macmillan, 1967, vol. 4, p. $418-420$.

10. Voir "Positivism and the Separation of Laws and Morals", loc. cit., pp. 62-72.

11. Ibid., pp. $72-78$.

12. Hart prétend en effet qu'une société esclavagiste pourrait rencontrer les exigences de ce "minimum de droit naturel » et des deux règles formelles qui l'accompagnent. Voir, ibid.,, p. 83.

13. Ce qui ne signifie pas par ailleurs que toute normativité, que ce soit celle du droit ou celle de la vie privée par exemple, soit de nature morale. Le droit comme d'autres champs de normativité comprend des règles qui sont purement conventionnelles.

14. Dans cette voie, on doit signaler que le refus de se prononcer par des autorités compétentes ou encore le maintien du statu quo demeure néanmoins une décision qui oriente normativement des actions et n'a rien à voir avec une position normativement neutre.

15. Je pense ici à la prise de position du célèbre philosophe du droit, Ronald Dworkin, défenseur contemporain d'une version « herméneutique " du naturalisme. Dans un article publié le 29 juin 1989 dans le New York Review of Books, quelques jours à peine avant la décision de la Cour suprême des Etats-Unis confirmant 
Ie droit de l'Etat du Missouri de refuser de défrayer les frais médicaux pour avortement, Dworkin mit en garde la plus haute cour américaine contre toute tentation de restreindre la portée de la décision Roe v. Wade de 1973 qui avait eu pour effet de décriminaliser partiellement l'avortement sur le territoire américain. Dworkin dans cet article défend la thèse que le droit à l'avortement est un "droit constitutionel ", c'est-à-dire un droit protégé par la constitution américaine. Il y critique aussi sévèrement au passage le mouvement pro-vie pour son attitude dogmatique et obscurantiste dans ce débat.

16. Qu'on pense seulement pour s'en convaincre à l'intérêt porté en théorie générale du droit depuis Austin à la compréhension de la spécificité de "l'obligation juridique".

17. Voir Georges A. Legault: La structure performative du langage juridique, Montréal: Les Presses de l'Université de Montréal, 1977, $522 \mathrm{p}$.

18. Voir p. 32 du texte de Legault.

19. Le concept de droit, op. cit., p. 251. Neil MacCormick dans son H.L.A. Hart, Stanford University Press, 1981, pp. 24-25 insiste sur la force de cet argument moral et sur l'importance qu'il faut lui accorder au sein du positivisme hartien.

20. Le concept de droit, op. cit., p. 251. 